AN. MED. INTERNA (Madrid) Vol. 19, N. ${ }^{\circ} 12$, pp. 644-648, 2002

\title{
Manejo diagnóstico-terapéutico del carcinoma de paratiroides
}

\author{
A. SILLERO SÁNCHEZ, Mª A. ATIENZA IGLESIAS* \\ Sección de Endocrinología y Nutrición y *Unidad de Medicina Familiar y Comunitaria. \\ Hospital Universitario Puerta del Mar. Cadiz.
}

\author{
DIAGNOSTIC-THERAPEUTIC MANAGEMENT OF PARATHYROID \\ CARCINOMA
}

\begin{abstract}
RESUMEN
El carcinoma de paratiroides es una neoplasia endocrina infrecuente, difícil de diagnosticar a priori, aunque existen datos clínicos y bioquímicos que lo sugieren, como las marcadas elevaciones en la calcemia y parathormona, la clínica de hipercalcemia severa o afectación de órganos diana y la palpación de una masa cervical. Se confirma por el análisis histopatológico, la invasión local o la existencia de metástasis ganglionares o a distancia. Su tratamiento es quirúrgico, mediante una resección en bloque, como única posibilidad para alcanzar su curación. Igualmente se indica la cirugía de las metástasis si son accesibles, con intención paliativa sobre la hipercalcemia. No son eficaces la radioterapia ni la quimioterapia para ello. En casos de hipercalcemia aguda o no susceptible de cirugía, se emplean fármacos antirresortivos (bifosfonatos) para su control. Se aconseja la realización de técnicas de localización previas a la reintervención por persistencia o recurrencia tumorales. Entre ellas, la ecografía cervical, TAC, RNM y la gammagrafía con Tc-99 sestamibi.
\end{abstract}

\begin{abstract}
Parathyroid carcinoma is an uncommon endocrine malignancy, with difficult diagnosis. There are several presenting clinical and biochemical features that suggest it: much higher serum calcium and PTH levels than parathyroid adenomas, symptoms of severe hypercalcemia, the classical target organs affected and a palpable neck mass. Pathologic findings, local invasion, lymph node and distant metastases prove the diagnosis. Initial surgical therapy (en bloc dissection) is the only chance for cure it. The management of recurrent and/or metastatic parathyroid carcinoma is also surgical, resulting in significant palliation from hypercalcemia, whereas radiation therapy and chemotherapy are not helpful. Bisphos phonates (drugs that inhibit bone resorption) control acute and chronic hypercalcemia when surgery is not effective or possible. Preoperative localization studies (cervical ultrasound, CT scan, MRI and sestamibi scan) are useful in patients with recurrent or persistent parathyroid can cer.
\end{abstract}

KEY WORDS: Parathyroid carcinoma. Hypercalcemia. Hyperparathy roidism. Diagnosis. Treatment.
PALABRAS CLAVE: Carcinoma de paratiroides. Hipercalcemia. Hiperparatiroidismo. Diagnóstico. Tratamiento.

\section{PROCESO DIAGNÓSTICO}

Existen una serie de características demográficas, clínicas y analíticas al diagnóstico del cuadro que sugieren la existencia de un CP y no otras causas de HPP (Tabla I):

-No existe predominio del CP por ningún sexo $(1-3,8)$ y la edad media es de 54 años (8), oscilando en las series más importantes entre los 44 y 55 años $(1-3,5,7,9,10)$.

- Suelen presentar la clínica típica de la hipercalcemia severa: debilidad (89\%), poliuria y/o polidipsia (22-38\%), artromialgias $(27 \%)$, pérdida de peso (17\%), anorexia, nauseas, vómitos, estreñimiento, dolores óseos, etc $(7,9)$. Tan sólo en un 2-7\% (7) no se aprecian síntomas, a diferencia del HPP donde la mayoría son asintomáticos (11) o tienen mínimos síntomas (12).

Trabajo aceptado: 17 de abril de 2002

Correspondencia: Álvaro Sillero Sánchez. Avenida de Andalucía, 26, $7^{\circ}$ A. 11008 Cádiz. 


\section{TABLA}

DIFERENCIAS CLÍNICO -ANALITICAS ENTRE EL HPP (HIPERPLASIA O ADENOM A) Y EL CP

\begin{tabular}{|c|c|c|}
\hline & HPP $^{11}$ & $C P$ \\
\hline $\begin{array}{l}\text { Sexo } \\
\text { Edad diagnóstico } \\
\text { Calcemia (mg/ dl) } \\
\text { PTH } \\
\text { Fosfatasa Alcalina }\end{array}$ & $\begin{array}{l}\text { Femenino } \\
6 \text { a década } \\
10,6 \\
\times 2 \\
\text { Límite superior } \\
\text { normalidad }\end{array}$ & $\begin{array}{l}\text { Indistinto } \\
54 \\
>14 \\
\times 5-10 \\
\times 3\end{array}$ \\
\hline $\begin{array}{l}\text { Crisis hipercalcémicas } \\
\text { Clínica } \\
\text { M asa palpable } \\
\text { Afectación } \\
\text { laríngeo recurrente }\end{array}$ & $\begin{array}{l}\text { Excepcional } \\
\text { Mínimos síntomas } \\
5-10 \% \\
\text { No }\end{array}$ & $\begin{array}{l}12-14 \% \\
\text { Frecuente } \\
50 \% \\
16 \%\end{array}$ \\
\hline $\begin{array}{l}\text { Nefrolitiasis } \\
\text { Afectación ósea } \\
\text { Afectación radiológica } \\
\text { Afectación renal } \\
\text { y ósea }\end{array}$ & $\begin{array}{l}<20 \% \\
10-25 \% \\
<5 \% \\
\text { Rara }\end{array}$ & $\begin{array}{l}32-70 \% \\
41-91 \% \\
\text { Frecuente } \\
32-53 \%\end{array}$ \\
\hline
\end{tabular}

HPP: Hiperparatiroidismo primario; CP: Carcinoma de Paratiroides

- Se palpa a nivel cervical una masa en el $50 \%$ de las ocasiones $(1,2,7)$, lo cual es muy infrecuente en el HPP.

-Presentan con frecuencia afectación de órganos diana. Así, la nefrolitiasis aparece en el 32-70\% de los CP $(3,5,7,10)$; los signos de afectación ósea en el 41-91\%, según el grado de afectación ósea considerado $(1,3,5,7,10)$ y la afectación conjunta renal y ósea (muy rara en el HPP) se detecta en un 32$53 \%$ de los CP $(1,3,5,7,9,10)$.

- La afectación del nervio laríngeo recurrente (sin otra patología previa que la justifique) es muy sugestiva de CP (1).

- La hipercalcemia del CP es mucho más severa (1-7) que la del HPP, cuya detección en la mayoría de las ocasiones es casual y leve (11). En el 65-75\% de las ocasiones la calcemia al diagnóstico es $>14 \mathrm{mg} / \mathrm{dl}(10,1-24 \mathrm{mg} / \mathrm{dl})(1,2,13)$. En un $12-14 \%(5,14)$ de las ocasiones se presenta como crisis hipercalcémica, siendo ello extremadamente raro en los adenomas paratiroideos.

- La PTH está elevada más de 5 veces por encima del límite superior de la normalidad (2), a diferencia del HPP donde no suele sobrepasar el doble del valor normal (11).

Con todos estos datos se puede sospechar a priori el diagnóstico de $\mathrm{CP}$, que sólo se confirma tras el análisis histopatológico. Un "segundo momento" que sugiere el diagnóstico es el intraoperatorio, donde las características macroscópicas del tumor deben indicar al cirujano la existencia de un CP, realizando una resección apropiada, como única posibilidad para alcanzar su curación.

El diagnóstico histopatológico presenta gran dificultad, incluso para anatomopatólogos expertos, y no es infrecuente el diagnóstico de CP a posteriori tras la recurrencia tumoral (local o a distancia) o de la hipercalcemia. Así, en una serie de cirujanos con experiencia en paratiroides, hasta el $86 \%$ de los $\mathrm{CP}$ no fueron apreciados inicialmente (8). Hoy existe una tendencia a la realización de técnicas de localización tumoral previas a la primera cirugía (gammagrafía con Tl-201, con Tc-99m-MIBI (15), ecografía, TAC, etc.), para facilitarla.
La punción con aguja fina y la biopsia no deben emplearse ante la sospecha de $\mathrm{CP}$, porque pueden provocar una diseminación del mismo por el tracto de punción (16). El diagnóstico intraoperatorio mediante cortes por congelación no es útil $(1,4,6)$.

Macroscópicamente el CP es de coloración grisáceoblanquecina, de consistencia firme, de aspecto ovoide y al corte lobulado (2), mientras que el adenoma es de coloración rojiza o parda brillante, blando y redondeado. En el 50-90\% $(2,17)$ está rodeado por una cápsula fibrosa y densa, que se adhiere fuertemente a los tejidos circundantes dificultando la extirpación del tumor. Por el contrario, el adenoma paratiroideo es fácilmente movilizado. La adhesión es la característica intraoperatoria que más debe hacer sospechar al cirujano la existencia de un CP (2). El CP puede infiltrar órganos vecinos (tiroides el más frecuente) y presentar afectación de ganglios linfáticos cervicales o metástasis a distancia al diagnóstico, lo cual, en los dos últimos supuestos, hace inequívoco el diagnóstico $(1,17)$. Sin embargo, ésto no está presente en la gran mayoría de los casos. Así, la afectación linfática sólo aparece en el 15-17\% (8,14), lo cual dificulta mucho el diagnóstico. Hasta en un $20 \%$ de los casos las características intraoperatorias son similares a las del adenoma (4).

Microscópicamente, Shantz y Castleman (2) establecieron unos criterios para diferenciar el adenoma del CP: 1) las células tumorales presentan un aspecto uniforme, organizándose con una arquitectura sólida, trabecular, que viene determinada por las prolongaciones a modo de septos al interior tumoral de la cápsula fibrosa $(80-95 \%$ de los casos) $(2,18)$, siempre que no esté provocado por una hemorragia antigua o cirugía previa; 2 ) presencia de mitosis $(45-81 \%)$ dentro de las células parenquimatosas, que es el criterio individual microscópico de más valor (2), aunque esto no ha sido confirmado en series posteriores (18-20). Tan sólo el 22\% de los $\mathrm{CP}$ presentaban un alto índice mitótico, por encima de lo que es habitual en lesiones benignas y pueden existir CP agresivos sin mitosis (18); 3 ) invasión capsular $(67 \%)$; 4) invasión vascular $(12-55 \%)$.

Ninguna de estas características es patognomónica de CP y todas ellas, incluida la invasión vascular, se han encontrado ocasionalmente en adenomas de paratiroides $(4,20)$. Cuantos más criterios reúna un tumor, más probable es que se trate de un CP. Si además se objetiva invasión local, metástasis ganglionares o a distancia, el diagnóstico se confirma.

Otros procedimientos empleados en los últimos tiempos para mejorar el diagnóstico, ofrecen pocas ventajas con respecto a los clásicos. Así, la microscopía electrónica no diferencia malignidad de benignidad $(17,21)$. El análisis del contenido nuclear y celular indica que el tamaño celular suele ser mayor en el CP, aunque se superpone al del adenoma y la relación núcleo/citoplasma es igualmente mayor en el CP. Las atipias nucleares en las células tumorales son sugestivas de CP (21), pero no patognomónicas, ya que se han descrito con diferente frecuencia en los adenomas $(1,2,18)$. La medida del contenido de ADN nuclear por citometría de flujo, (suele ser mayor en el CP que en el adenoma (20)), puede tener valor en el diagnóstico del CP en casos dudosos (18), aunque August y cols. (22) señalan que hasta en el $44 \%$ de los mismos aparecen falsos negativos y no diferencia la malignidad de la benignidad como parámetro único, aunque 
sí es útil en la predicción del comportamiento clínico del CP (22). El patrón aneuploide de ADN es más frecuente (22) en el CP, aunque también se describe con cierta frecuencia en los adenomas $(6,22-24)$.

\section{TÉCNICAS DE LOCALIZACIÓN}

Deben practicarse antes de realizar reintervenciones por recurrencia local o a distancia o persistencia tumoral (se recomiendan al menos dos, que deben ser concordantes) (32). Hoy en día, con la mejoría de su sensibilidad, se suelen realizar rutinariamente cuando se detecta una hipercalcemia o elevación de la PTH en su seguimiento. Debe comenzarse por la palpación cervical detenida, pues es el sitio de recurrencia más precoz y frecuente y hasta el $45 \%$ de las recurrencias son palpables (37). Entre los métodos de localización se encuentran los morfológicos como la ecografía cervical, para la detección de pequeños tumores cervicales, especialmente los adyacentes al tiroides o intratiroideos (38). Las características ecográficas del CP son: masas hipoecoicas, grandes, de ecogenicidad no homogénea, con contornos lobulados y con una relación profundidad/anchura $\geq 1$ en el $95 \%$ de los casos. De este modo puede diferenciarlos del adenoma de paratiroides, que es más pequeño, hipoecoico, de ecogenicidad homogénea, bordes lisos y relación profundidad/anchura <1 en el $95 \%$ de los casos (39). Igualmente la detección ecográfica de invasión de estructuras adyacentes, orienta el diagnóstico hacia el CP (40). Por tanto, la ecografía no sólo es útil en la localización prequirúrgica, sino también en la diferenciación entre adenoma y carcinoma (39). Otras técnicas morfológicas son la radiografía de tórax, la TAC, la RNM de tórax y abdomen, útiles en la detección de metástasis en estas localizaciones, la tomografía por emisión de positrones y las radiografías óseas. Entre las pruebas morfofuncionales, la gammagrafía con Tc-99m sestamibi es la más empleada, localiza la recurrencia e identifica metástasis locales y a distancia, no diferenciando benignidad de malignidad $(15,41,42)$. Incluso, se ha empleado intraoperatoriamente para la localización del tejido paratiroideo alterado (43). La PET se ha empleado cuando el resto de técnicas son equívocas o negativas y no parece tener utilidad (32).

Si no se llega al diagnóstico con las técnicas no invasivas, o no existe concordancia entre sus hallazgos, se han empleado otras técnicas invasivas de localización, como la cateterización venosa selectiva prequirúrgica para PTH en cuello, mediastino, venas hepática, renal, cava inferior e iliaca y la arteriografía (44), para identificar múltiples sitios de recurrencia y tumor en localizaciones a distancia.

La sensibilidad de la ecografía, TAC, RNM, MIBI y cateterización venosa selectiva descrita es del 69, 67, 93, 79 y $83 \%$ respectivamente $(32)$.

Si no se encuentra el tumor o la recurrencia con las diferentes técnicas de localización, se maneja con tratamiento médico hasta que se localice y si no se controla la hipercalcemia, se reexploran el cuello y el mediastino para hallar enfermedad recurrente, aunque si ello se repite con frecuencia las adherencias dificultarían posteriores localizaciones.

\section{TRATAMIENTO}

Debe estar dirigido a la extirpación del tumor primario, de sus posibles recurrencias o metástasis y al control de la hipercalcemia, que es la principal causa de morbimortalidad.

\section{CIRUGÍA}

El tratamiento de elección es el quirúrgico, tanto en primera instancia, como en las sucesivas recurrencias y metástasis accesibles con fines paliativos sobre la hipercalcemia. En el $89 \%$ de los casos es el único tratamiento realizado (8) y es la única posibilidad de alcanzar la curación. Hay que explorar las 4 paratiroides por la posibilidad de que coexista un adenoma o hiperplasia en las demás glándulas (25-30).

1. Debe realizarse una resección completa en bloque de todo el tumor posible en la primera operación (cuando la probabilidad de invasión local y a distancia es baja $(1,3,5,6,20,31))$, junto con el istmo y el lóbulo tiroideo ipsilateral, extirpación de cualquier tejido contiguo al que se adhiera el tumor (timo, músculos cervicales o tejido conectivo), esqueletización de la traquea, extirpación de los ganglios linfáticos paratraqueales, los traqueoesofágicos y mediastínicos superiores, así como aquéllos que se encuentren aumentados de tamaño. Si el nervio laríngeo recurrente está envuelto o adherido por el tumor hay que extirparlo $(3,7,14)$. Es muy importante evitar la ruptura de la cápsula siempre que se sospeche un $\mathrm{CP}$, pues favorecería la diseminación y recurrencias locales del tumor, impidiendo su curación. La disección cervical radical extensa sólo está indicada en casos de invasión local extensa clara en el momento de la cirugía, existencia de múltiples ganglios cervicales aumentados de tamaño o en reintervenciones en las que las cicatrices impiden delimitar la extensión del tumor $(2,4,5)$. No debe realizarse de forma profiláctica, pues no mejora la supervivencia.

A pesar de estas recomendaciones y debido al alto porcentaje de CP no diagnosticados a priori, hasta en el $60 \%$ de los casos la cirugía inicial fue parcial y sólo en el $13 \%$ fue completa (8).

En el postoperatorio inmediato hay que monitorizar la calcemia, ya que los pacientes en los que se elimina todo el tumor pueden desarrollar hipocalcemia severa por el síndrome del hueso hambriento, precisando grandes dosis de calcio iv y calcitriol oral, disminuyendo las necesidades con el tiempo. Posteriormente se deben realizar determinaciones de calcio y PTH cada 3 meses. La hipercalcemia o hiperparatiroidismo(su elevación es más precoz) (32) persistentes tras la cirugía del CP suelen indicar la presencia de una extirpación incompleta o de metástasis. La elevación durante el seguimiento de la calcemia es indicativa de recurrencia tumoral y suele preceder a la evidencia física de enfermedad recurrente. El seguimiento debe realizarse de por vida, para alcanzar un diagnóstico precoz de las recurrencias.

2. Si el diagnóstico se hace a posteriori por el análisis histopatológico de la pieza tumoral y existe invasión capsular o vascular extensa, el paciente permanece hipercalcémico o las técnicas de localización muestran tumor residual, debe realizarse una nueva exploración quirúrgica cervical, y resecar las estructuras adyacentes a donde estaba localizado el tumor, pues el lecho quirúrgico es el lugar de mayor probabilidad de recurrencia, extirpando igualmente el hemitiroides (9). Si al reexplorar la pieza quirúrgica el CP tenía un comportamiento muy agresivo, pueden existir ya metástasis linfáticas o a distancia. Si no ocurre esto, suelen ser $\mathrm{CP}$ menos agresivos y en ocasiones la simple resección tumoral realizada puede ser curativa, no haciendo falta reintervenir salvo que en el seguimiento se detecten elevaciones de la calcemia y/o PTH. 
3. En el caso de que se detecte una recurrencia tumoral local o a distancia, el tratamiento es también quirúrgico, resecando ampliamente la zona afecta e incluyendo los ganglios linfáticos regionales y otras estructuras envueltas a nivel cervical. Esto se realizará tantas veces como sea preciso (6) y accesibles sean las recurrencias, como tratamiento paliativo $(1,3,10)$, ya que de esta forma se facilita el manejo de la hipercalcemia asociada, se mitigan los síntomas relacionados con la misma hasta en el $86 \%$ de las ocasiones (32) y se aumenta la supervivencia. En muy contadas ocasiones la resección de una primera recurrencia es curativa, describiéndose periodos libres de recurrencia de hasta 7 años (33). Estas reintervenciones consiguen la normocalcemia en el $68-75 \%$ de los casos si es la segunda cirugía y en el $55 \%$ en la tercera $(7,32)$. El único factor significativo que predice la obtención de la normocalcemia tras la reintervención es la calcemia prequirúrgica (32). Se ha descrito una importante morbilidad asociada con la reintervención, principalmente debido a parálisis del nervio laríngeo recurrente (17\%) (32).

En el caso de las metástasis a distancia pulmonares, se realiza la metastasectomía de todas las posibles, incluso si son bilaterales o múltiples (hasta 34 en una sóla intervención (31)), alcanzándose la normocalcemia durante periodos de 1-3 años $(6,10)$ y hasta 10 años (34). En general se obtienen peores resultados que a nivel cervical en el control de la hipercalcemia, aunque facilita el control posterior de la misma con fármacos $(3,5-8,10,31,35)$. En ocasiones se requieren múltiples reintervenciones. Para su indicación debe existir control de la enfermedad local a nivel cervical, no otras metástasis concurrentes en otras localizaciones y una historia natural indolente del tumor. Un intervalo libre de enfermedad prolongado entre la primera cirugía y la aparición de metástasis pulmonares, se correlaciona con una buena supervivencia tras la metastasectomía (31). A nivel hepático se puede realizar de la misma manera la metastasectomía cuando son accesibles. Se ha descrito la resección guiada por RNM intraoperatoria de una metástasis intracraneal con éxito (36).

\section{RADIOTERAPIA}

Se emplea junto con la cirugía en el $6 \%$ de los casos. Sólo en el $1 \%$ de los casos se emplea como único tratamiento (8). Los intentos de empleo de la radioterapia para el control del volumen tumoral y su producción hormonal han sido inefectivos en la mayoría de las ocasiones $(1-4,7)$ y no ha demostrado que mejore la supervivencia (3). Sólo en casos muy puntuales $(7,45,46)$ se ha descrito su utilidad, como en la radiación cervical preventiva de recurrencias locales (si existe alto riesgo de ello por ruptura de la cápsula o cirugía incompleta) $(20,22,45)$, o de forma paliativa si no es posible la cirugía (47).

\section{TRATAMIENTO QUIMIOTERÁPICO Y FARMACOLÓGICO}

No hay datos favorables al empleo de la quimioterapia en el CP, salvo excepciones (48-50) y no se ha demostrado que aumente la supervivencia (3). No hay una pauta de tratamiento única y se han empleado múltiples combinaciones de fár$\operatorname{macos}(7,10,25,45)$.

Cuando el tumor no es susceptible de resección quirúrgica, se puede alcanzar una larga supervivencia controlando la hipercalcemia, con una adecuada hidratación y aumentando la calciuria con diuréticos del asa, lo cual debido a las elevadas cifras de PTH y a la intensidad de la resorción ósea no suele conseguirse. Por ello, se precisan otra serie de fármacos para el control de la hipercalcemia. Los más usados hoy en día son los bifosfonatos, que debido a su acción antirresortiva disminuyen, al menos transitoriamente, la calcemia. Dentro de este grupo se han empleado el clodronato $(51,52)$, el etidronato $(53,54)$ y el pamidronato $(6,31,55,56)$, que se han utilizado también para el control de la hipercalcemia previo a la cirugía.

Otros fármacos antirresortivos (mitramicina (3), calcitonina $(3,37,52,57)$, sóla o asociada a corticoides (58) y nitrato de galio $(10,59))$ han sido empleados previamente a la aparición de los bifosfonatos, desechándose por producir mayores efectos adversos.

Recientemente se han ensayado nuevos fármacos para el control de la hipercalcemia tumoral, como el octreótide; los agonistas del receptor de la PTH, que disminuyen los niveles de PTH y calcio de forma aguda y mantienen estable la calcemia al menos 2 años, sin aparición de efectos adversos (60); y la inmunización con péptidos PTH humanos y bovinos, que inducen la producción de anticuerpos contra la PTH, bloqueando su unión con los receptores y normalizando la calcemia durante 6 meses, sin efectos adversos relevantes $(61,62)$.

\section{Bibliografía}

1. Holmes EC, Morton DL, Ketcham AS. Parathyroid carcinoma: A collective review. Ann Surg 1969; 169: 631-40.

2. Shantz A, Castleman B. Parathyroid carcinoma: A study of 70 cases. Cancer 1973; 31: 600-5.

3. Shane E, Bilezikian J. Parathyroid carcinoma: a review of 62 patients. Endocr Rev 1982; 3: 218-26.

4. Cohn K, Silverman M, Corrado J, Sedgewick C. Parathyroid carcinoma: The Lahey Clinic experience. Surgery 1985; 98: 1095-1110.

5. Wang C, Gaz R. Natural history of parathyroid carcinoma: diagnosis, treatment, and results. Am J Surg 1985; 149: 522-7.

6. Sandelin K, Thompson NW, Bondeson L. Metastatic parathyroid carcinoma: dilemmas in management. Surgery 1991; 110: 978-86.

7. Wynne A, Hereden J, Carney J, Fitzpatrick LA. Parathyroid carcinoma: clinical and pathological features in 43 patients. Medicine 1992; 71: 197-205.
8. Hundahl SA, Fleming ID, Fremgen AM, Menck HR. Two hundred eighty-six cases of parathyroid carcinoma treated in U.S. between 19851995: a National Cancer Data Base Report. Cancer 1999; 86: 538-44.

9. Shortell CK, Andrus CH, Phillips CE Jr, Schwartz SI. Carcinoma of the parathyroid gland: A 30-year experience. Surgery 1991; 110: 704-8.

10. Vetto JT, Brennan MF, Woodruf J, Burt M. Parathyroid carcinoma: diagnosis and clinical history. Surgery 1993; 114: 882-92.

11. Silverberg SJ, Shane E, Jacobs TP, Siris E, Bilezikian JP. A 10-year prospective study of primary hyperparathyroidism with or without parathyroid surgery. N Engl J Med 1999; 341: 1249-55.

12. Burney RE, Jones KR, Christy B, Thompson NW. Health status improvement after surgical correction of primary hyperparathyroidism in patients with high and low preoperative calcium levels. Surgery 1999; 125: $608-14$ 
13. Mallette L, Bilezikian J, Ketcham A, Aurbach G. Parathyroid carcinoma in familiar hyperparathyroidism. Am J Med 1974; 57: 642-8.

14. Obara T, Fujimoto Y. Diagnosis and treatment of patients with parathyroid carcinoma: an update and review. World J Surg 1991; 15: 738-744

15. Kitapci MT, Tastekin G, Turgut M, et al. Preoperative localization of parathyroid carcinoma using Tc-99m MIBI. Clin Nucl Med 1993; 18: 217-9.

16. Spinelli C, Bonadio AG, Berti P, Materazzi G, Miccoli P. Cutaneus spreading of parathyroid carcinoma after fine needle aspiration cytology. J Endocrinol Invest 2000; 23: 255-7.

17. Smith JF, Coombs RRH. Histological diagnosis of carcinoma of the parathyroid gland. J Clin Pathol 1984; 37: 1370-8.

18. Bondeson L, Sandelin K, Grimelius L. Histopathological variables and DNA cytometry in parathyroid carcinoma. Am J Surg Pathol 1993; 17: 820-9.

19. Snover DC, Foucar K. Mitotic activity in benign parathyroid disease. Am J Clin Pathol 1984; 37: 1370-8.

20. Levin KE, Galante M, Clarck OH. Parathyroid carcinoma versus parathyroid adenoma in patients with profound hypercalcemia. Surgery 1987; 101: 649-60.

21. Altenähr E, Salger W. Light and electron microscopy of parathyroid carcinoma. Virchows Arch 1973; 360: 107-22.

22. August DA, Flynn SD, Jones MA, Bagwell CB, Kinder BK. Parathyroid carcinoma: the relationship of nuclear DNA content to clinical outcome. Surgery 1993; 113: 290-6.

23. Bowlby LS, DeBault LE, Abraham SR. Flow cytometric DNA analysis of parathyroid glands. Am J Pathol 1987; 128: 338-44.

24. Mallette LE. DNA quantitation in the study of parathyroid lesions. A review. Am J Clin Pathol 1992; 98: 305-11.

25. Golden A, Canary JL, Kerwin DM. Concurrence of hyperplasia and neoplasia of the parathyroids glands. Am J Med 1965; 38: 562-78.

26. Kramer W. Association of parathyroid hyperplasia with neoplasia. Am J Clin Pathol 1970; 53: 275-83.

27. Murayama T, Kawabe K, Tagami M. A case of parathyroid carcinoma concurred with hyperplasia: an electron microscopic study. J Urol 1977; 118: 126-7.

28. Aldinger KA, Hickey RC, Ibanez ML, Samaan NA. Parathyroid carcinoma: a clinical study of seven cases of functioning and two cases of nonfunctioning parathyroid cancer. Cancer 1982; 49: 388-97.

29. Haghighi P, Astarita RW, Wepsic HT, Wolf PL. Concurrent primary parathyroid hyperplasia and parathyroid carcinoma. Arch Pathol Lab Med 1983; 107: 349-50.

30. Shapiro DM, Recant W, Hemmati M, Mazzone T, Evans RH. Synchronous occurrence of parathyroid carcinoma and adenoma in an elderly woman. Surgery 1989; 106: 929-33.

31. Obara T, Okamoto T, Ito Y, et al. Surgical and medical management of patients with pulmonary metastasis from parathyroid carcinoma. Surgery 1993; 114: 1040-9.

32. Kebebew E, Arici C, Duh QY, Clark OH. Localization and reoperation for persistent and recurrent parathyroid carcinoma. Arch Surg 2001; 136: 878-85.

33. Fraker DL, Travis WD, Merendino JJ Jr, et al. Locally recurrent parathyroid neoplasms as a cause for recurrent and persistent hyperparathyroidism. Ann Surg 1991; 213: 58-65.

34. Davies DR, Dent CE, Ives DR. Successful removal of single metastasis in recurrent parathyroid carcinoma. Br Med J 1973; 1: 397-8.

35. Favia G, Lumachi F, Polistina F, D'Amico DF. Parathyroid carcinoma: sixteen new cases and suggestions for correct management. World J Surg 1998; 22: 1225-30.

36. Tyler D 3rd, Mandybur G, Dhillon G, Fratkin J. Intracranial metastatic parathyroid carcinoma: a case report. Neurosurgery 2001; 48: 937-9.

37. Dubost C, Jehanno C, Lavergne A, Charpentier Y. Successful resection of intrathoracic metastases from two patients with parathyroid carcinoma. World J Surg 1984; 8: 547-51.

38. Daly BD, Coffrey SL, Behan M. Ultrasonic appearances of parathyroid carcinoma. Br J Radiol 1989; 62: 1077-9.

39. Hara H, Igarashi A, Yano Y, et al. Ultrasonographic features of parathyroid carcinoma. Endocr J 2001; 48: 213-7.
40. Edmonson GR, Charboneau JW, James EM, Reading CC, Grant CS Parathyroid carcinoma: high frequency sonographic features. Radiology 1986; 151: 65-7.

41. Majors JD, Bruke GJ, Mansberger AR Jr. Technetium Tc-99m-sestamibi scan for localizing abnormal parathyroid glands after previous neck operations: preliminary experience in reoperative cases. South Med J 1995; 88: 327-30.

42. Cordeiro AC, Montenegro FL, Kulcsar MA, et al. Parathyroid carcinoma. Am J Surg 1998; 175: 52-5.

43. Martínez DA, King DR, Romshe C. Intraoperative identification of parathyroid gland pathology: a new approach. J Pediatr Surg 1995; 30: 1306-9.

44. Krudy AG, Doppman JL, Marx SJ, Brennan MF, Spiegel A, Aurbach GD. Radiographic findings in recurrent parathyroid carcinoma. Radiology 1982; 142: 625-9.

45. Chow E, Tsang RW, Brierley JD, Filice S. Parathyroid carcinoma. The Princess Margaret Hospital experience. Int J Radiation Oncology Biol Phys 1998; 41: 569-72.

46. Rasmuson T, Kristoffersson A, Boquist L. Positive effect of radiotherapy and surgery on hormonally active pulmonary metastases of primary parathyroid carcinoma. Eur J Endocrinol 2000; 143: 749-54.

47. McKeown PP, McGarity WC, Sewell CW. Carcinoma of the parathyroid glands: Is it overdiagnosed? A report of three cases. Am J Surg 1984; 147: 292-8.

48. Bukowski R, Sheeler L, Cunningham J, Esselstyn C. Successful combination chemoterapy for metastatic parathyroid carcinoma. Arch Int Med 1984; 144: 399-400.

49. Calandra DB, Chejfec G, Foy BK, Lawrence AM, Paloyan E. Parathyroid carcinoma: Biochemical and pathologic response to DTIC. Surgery 1984; 96: 1132-7.

50. Chahinian A, Holland J, Nieburgs H, Marinescu A, Seller S, Kirschner P. Metastatic nonfunctioning parathyroid carcinoma: ultrastructural evidence of secretory granules and response to chemoterapy. Am J Med Sci $1981 ; 282$ : 80-4.

51. Shane E, Jacobs T, Siris E, et al. Therapy of hypercalcemia due to parathyroid carcinoma with intravenous dichloromethylene diphosphonate. Am J Med 1982; 72: 939-44.

52. Jungst D. Disodium clodronate is effective in management of severe hypercalcemia caused by parathyroid carcinoma. Lancet 1984; 1: 1043.

53. Ralston SH, Alzaid AA, Gardner MD, Boyle IT. Treatment of cancerassociated hypercalcemia with combined aminohydroxypropylidene diphosphonate and calcitonin. Br Med J 1986; 292: 1549-50.

54. Jacobs T, Gordon A, Gundberg C, et al. Neoplastic hypercalcemia: physiologic response to intravenous etidronate. Am J Med 1987; 82: 42-52.

55. Vega EM, Mautalen CA. Intravenous pamidronic acid in hypercalcemia due to parathyroid carcinoma. Medicina 1991; 51: 106-10.

56. Meier DE, Zinder WH 3rd, Dickson BA, Margraf LR, Guzzetta PC Jr. Parathyroid carcinoma in a child. J Pediatr Surg 1999; 34: 606-8.

57. Trigonis C, Cedermark B, Willems J, Hamberger B, Granberg PO. Parathyroid carcinoma. Problems in diagnosis and treatment. Clin Oncol 1984; 10: 11-9.

58. Au WY. Calcitonin treatment of hypercalcemia due to parathyroid carcinoma: Synergistic effect of prednisone on longterm treatment of hypercalcemia. Arch Intern Med 1975; 135: 1594-7.

59. Warrell RP Jr, Isaacs M, Alcock NW, Bockman RS. Gallium nitrate for treatment of refractory hypercalcemia from parathyroid carcinoma. Ann Intern Med 1987; 197: 683-6.

60. Collins MT, Skarulis MC, Bilezikian JP, Silverberg SJ, Spiegel AM, Marx SJ. Treatment of hypercalcemia secondary to parathyroid carcinoma with a novel calcimimetic agent. J Clin Endocrinol Metab 1998; 83: 1083-8.

61. Bradwell AR, Harvey TC. Control of hypercalcaemia of parathyroid carcinoma by inmunisation. Lancet 1999; 353: 370-3.

62. Shane E. Parathyroid carcinoma. J Clin Endocrinol Metab 2001; 86: 485-93. 